\title{
Rapport annuel 2009
}

Durant l'exercice écoulé, la Caisse de secours des médecins suisses a accordé son soutien à 31 médecins tombés malgré eux dans le besoin (ils étaient 40 en 2008) ainsi qu'aux proches et aux descendants de médecins décédés.

Pendant la même période, la Caisse a cessé d'apporter son aide à 13 personnes. Toujours en 2009, quatre nouveaux cas sont venus s'ajouter à la liste des bénéficiaires.

Les produits ont résulté de recettes provenant de dons pour un montant de $122588 \mathrm{CHF}$ (moyenne des dix dernières années: 156256 CHF) ainsi que des intérêts et des dividendes du capital de la fondation, à hauteur de $68224 \mathrm{CHF}$.

Concernant les charges, les contributions de soutien se sont élevées à 308000 CHF (moyenne des dix dernières années: 319503 CHF). Les dépenses administratives se sont montées à 45195 CHF. Les trois postes les plus importants en sont la publication du rapport annuel dans le BMS et l'envoi, en décembre, de la lettre individuelle aux donateurs membres de la FMH pour un montant de $16783 \mathrm{CHF}$, les frais de gestion de la fortune, de $16314 \mathrm{CHF}$ et les frais comptables de la Banque La Roche, de 5380 CHF.

Les années où les cours étaient favorables, la différence entre les recettes provenant de dons, d'intérêts et de dividendes du capital et les charges était compensée par les gains boursiers. L'année dernière, aucun gain boursier n'a pu être réalisé. Cette évolution défavorable a entraîné une diminution du capital de la fondation, qui a ainsi passé à $3092600 \mathrm{CHF}$ (2008: 3163274 CHF).

La fortune de la fondation est constituée par un mélange d'obligations et d'actions satisfaisant aux prescriptions de la prévoyance professionnelle (LPP).
Le compte d'exploitation, le bilan et la gestion de la fortune sont révisés chaque année par l'autorité de surveillance du Département de justice du canton de Bâle-Ville.

Les dons individuels inférieurs à $500 \mathrm{CHF}$ forment l'essentiel du capital de notre fondation. Afin de réduire nos frais administratifs au strict minimum, nous renonçons à remercier personnellement les donateurs. Qu'ils veuillent trouver ici l'expression de notre vive gratitude.

Les comptes annuels de la Caisse de secours ont été révisés par la fiduciaire Axt Treuhand, laquelle a constaté que la comptabilité était tenue dans les règles. Nous la remercions vivement de son travail. Pour des informations détaillées, le lecteur se référera au compte de résultat et au bilan publiés ci-après.

Je remercie chaleureusement tous les membres de la Commission pour le travail qu'ils ont accompli à titre bénévole durant l'exercice passé sous revue. J'adresse également mes sincères remerciements à Messieurs J. Kobler et W. Mahrer de la Banque La Roche \& Co pour la tenue de la comptabilité et la gestion de la fortune.

La commission de fondation de la Caisse de secours des médecins suisses:

Dr U. Leibundgut, président

Mme le Dr S. Stöhr

Dr U. Lüscher, trésorier

Compte postal de la Caisse de secours des médecins suisses: 40-644-3 Basel 


\section{Comptes annuels 2009 (en francs)}

Bilan au 31 décembre 2009

Comptes Banque La Roche

Compte Postal

Débiteur impôt anticipé

Titres

Compte de régularisation des passifs

Provision sur titres

Fortune au 31 décembre 2009

Dr. J. Ambühl-Stiftung

Dr. J. Genhard-Stiftung

Dr. J. B. Wutz-Stiftung

Caisse de secours au $1^{\mathrm{er}}$ janvier

Changement de l'exercice

Compte d'exploitation pour 2009

Dons

Revenu sur capital

Ajustement provision sur titres

Ajustement change

Profits sur titres (non réal.)

Contributions

Frais de banque et d'administration

Intérêts débiteurs

Pertes sur titres (réal.)

Changement de l'exercice
Actifs

Passifs

75583.83

147352.12

16245.05

2853419.25

19983.70

852000.00

90000.00

20000.00

19450.75

2094275.50

2223726.25

$-3109.70$

3092600.25

3092600.25

Produits

122588.00

68224.10

68000.00

1745.50

157268.05

308000.00

45195.20

17.05

67723.10

$-3109.70$ 